\title{
Conclusions
}

Activities are an important means for residents of long term care facilities for obtaining pleasure and giving a meaningful structure to the day. Future lessons can be learned from the adjustments that had to be made in the range of activities offered during the visitor-ban.

\section{8 - Person-centred infection prevention and control during a pandemic: The Dementia Isolation Toolkit}

\begin{abstract}
Authors: Andrea Iaboni, Hannah Quirt, Steven Stewart, Alisa Grigorovich, Claudia Barned, Kevin Rodrigues, Pia Kontos, Charlene Chu, Arlene Astell, Katia Engell, Colleen Maxwell, Julia Kirkham, Kathleen Bingham, Alastair Flint
\end{abstract}

Objectives: People working in long-term care homes (LTCH) face ethical dilemmas about how to minimize the risk of spread of COVID-19, while also minimizing psychological hardship and other harms of infection control measures on residents. The Dementia Isolation Toolkit (www.dementiaisolationtoolkit.com; DIT) was developed to address the gap in ethical guidance for LTCH on how to safely and effectively isolate people with dementia while supporting the personhood and well-being of residents. In this presentation, we will present the DIT and report on the results of a survey of LTCH staff in Ontario, Canada on their experiences isolating residents in LTCH and the use of the DIT in supporting person-centred isolation care.

Methods: A link to an online survey was distributed to LTCH staff through provincial organizations and agencies as well as through social media and the DIT website. Inclusion criteria were LTCH staff working on-site at a LTCH since March 1, 2020, who had direct or indirect experience with the isolation/quarantine of LTCH residents. Results were summarized descriptively.

Results: A broad sample of LTCH staff ( $n=207)$ participated in the survey, most of whom had experienced an outbreak in their LTCH. Dementia (96\%) was the most important barrier to implementation of infection control measures in $\mathrm{LTCH}$, followed by staff distress about the effects of isolation on residents (61\%). Important facilitators for isolation included delivery of 1:1 activities in the resident's room (81\%) and designating essential caregivers to provide support (67\%), while inadequate staffing levels were reported as a barrier (55\%). $65 \%$ of respondents indicated some familiarity with the DIT, and of those who had used the toolkit, $62 \%$ found it helpful in supporting isolation care, particularly in developing care plans and making and communicating decisions. Of those who had used the DIT, 48\% found it fairly or very helpful at reducing their level of distress.

Conclusions: Isolation as an infection control and prevention (ICP) measure in LTCH environments can be harmful to residents and create moral distress in staff. ICP guidance and support of LTCH needs to address how to minimize these harms by providing dementia-specific guidance such as in the DIT. 